\title{
SHEAF COHOMOLOGY WITH BOUNDS AND BOUNDED HOLOMORPHIC FUNCTIONS
}

\author{
YUM-TONG SIU
}

Suppose $U$ is the unit disc in $C$. For $0<r<1 Q_{r}$ (or simply $Q$ ) is the annulus $\{z \in U|| z \mid>r\}$. A subvariety $V$ of pure codimension 1 in $U^{N}$ is called a Rudin subvariety if for some $r V \cap Q^{N}=\varnothing$. A Rudin subvariety is called a special Rudin subvariety if there is $\delta>0$ such that, for $1 \leqq k \leqq N,\left(z^{\prime}, a_{i}, z^{\prime \prime}\right) \in\left(Q^{k-1} \times U \times Q^{N-k}\right) \cap V, i=1,2$, and $a_{1} \neq a_{2}$, we have $\left|a_{1}-a_{2}\right| \geqq \delta$. If a holomorphic function $f$ generates the ideal-sheaf of its zero-set $E$, then we write $Z(f)=E$. The Banach space of all bounded holomorphic functions on a reduced complex space $X$ under the sup norm is denoted by $H^{\infty}(X)$ and the norm of $f \in H^{\infty}(X)$ is denoted by $\|f\|_{X}$. The following two theorems were proved by W. Rudin [2] and H. Alexander [1] respectively.

THEOREM 1. If $V$ is a Rudin subvariety, then there is $f \in H^{\infty}\left(U^{N}\right)$ such that $Z(f)=V$.

Theorem 2. If $V$ is a special Rudin subvariety, then there is a bounded linear map from $H^{\infty}(V)$ to $H^{\infty}\left(U^{N}\right)$ which extends every bounded holomorphic function on $V$ to one on $U^{N}$.

Cartan's Theorem B implies that an analytic hypersurface of a polydisc is the zero-set of a holomorphic function and that every holomorphic function on the hypersurface is induced by a holomorphic function on the polydisc. One can expect that some Theorem $B$ with bounds would easily yield the above two theorems. In this note we prove a simple theorem on sheaf cohomology with bounds (Theorem 3 below) which can imply Theorems 1 and 2. This gives us more perspective proofs of these two theorems.

Suppose $X$ is a reduced complex space and $\Theta$ is the structure-sheaf of $X \times U^{N}$. Let $W_{k}=X \times U^{k-1} \times Q \times U^{N-k}, 1 \leqq k \leqq N$, and $\mathfrak{W}=\left\{W_{k}\right\}$. For $\nu \geqq 0$ and $1 \leqq i_{0}, \cdots, i_{\nu} \leqq N W_{i_{0} \cdots i_{\nu}}$ denotes $W_{i_{0}} \cap \cdots \cap W_{i_{\nu}}$. If $f \in C^{\nu}(\mathfrak{W}, \mathcal{O})$, then $f_{i_{0} \ldots i_{\nu}} \in \Gamma\left(W_{i_{0} \cdots i_{\nu}}, \mathcal{O}\right)$ denotes the value of $f$ at the simplex $\left(W_{i_{0}}, \cdots, W_{i_{\nu}}\right)$ of the nerve of $\mathfrak{W}$. Let $\rho=2 /(1-r)$ and for $1 \leqq \nu<N$ let

$$
\sigma_{\nu}=\sum_{\mu=1}^{N}\left(\begin{array}{l}
N \\
\mu
\end{array}\right)(\nu+1)^{\mu-1} \rho^{\mu}
$$

Received by the editors March 11, 1968. 
Lемма 1. Suppose $f$ is a bounded holomorphic function on $X \times Q$ whose absolute value is bounded by a positive number $K$. Suppose for $w \in X f(w, z)=\sum_{\mu=-\infty}^{\infty} h_{\mu}(w) z^{\mu}$ is the Laurent series expansion of $f$ in $z$ (where $z$ is the coordinate function of $Q$ ). Let $g(w, z)=\sum_{\mu=0}^{\infty} h_{\mu}(w) z^{\mu}$ on $X \times Q$. Then $\|g\|_{X \times Q} \leqq \rho K$.

Proof. Fix $(w, z) \in X \times Q$. Choose arbitrarily two positive numbers $a$ and $b$ such that $r<a<|z|<b<1$. We need only prove that $|g(w, z)|$ $\leqq 2 b K /(b-a)$, because the result follows then from letting $a \rightarrow r$ and $b \rightarrow 1$.

Case (i). $|z| \leqq(a+b) / 2$. Then $|\zeta-z| \geqq(b-a) / 2$ for $|\zeta|=b$.

$$
|g(w, z)|=\left|\frac{1}{2 \pi i} \int_{|\zeta|=b} \frac{f(w, \zeta)}{\zeta-z} d \zeta\right| \leqq \frac{2 b}{b-a} K .
$$

Case (ii). $|z| \geqq(a+b) / 2$. Then $|\zeta-z| \geqq(b-a) / 2$ for $|\zeta|=a$.

$$
|f(w, z)-g(w, z)|=\left|\frac{-1}{2 \pi i} \int_{|\zeta|=a} \frac{f(w, \zeta)}{\zeta-z} d \zeta\right| \leqq \frac{2 a}{b-a} K .
$$

Hence $|g(w, z)| \leqq 2 b K /(b-a)$.

Q.E.D.

TheOREM 3. For $1 \leqq \nu<N$ there exists a linear map $\phi_{\nu}: B^{\nu}(\mathfrak{B}, \mathcal{O})$ $\rightarrow C^{\nu-1}(\mathfrak{W}, \mathcal{O})$ over the ring of all holomorphic functions on $X$ such that

(i) $\delta \phi_{\nu}=$ the identity map on $B^{\nu}(\mathfrak{W}, \mathcal{O})$, and

(ii) if $f \in B^{\nu}(\mathfrak{W}, \mathcal{O})$ and $\left\|f_{i_{0} \cdots i_{\nu}}\right\|_{W i_{0} \cdots i_{\nu}} \leqq K$ for $1 \leqq i_{0}, \cdots, i_{\nu} \leqq N$, then $\left\|\left(\phi_{\nu}(f)\right)_{i_{0} \cdots i_{\nu-1}}\right\|_{W i_{0} \cdots i_{\nu-1}} \leqq \sigma_{\nu} K$ for $1 \leqq i_{0}, \cdots, i_{\nu-1} \leqq N$.

Proof. First we define for $1 \leqq i \leqq N$ and $0 \leqq \nu<N$ a linear map $e_{i}: C^{\nu}(\mathfrak{W}, \mathcal{O}) \rightarrow C^{\nu}(\mathfrak{W}, \mathcal{O})$ over the ring of all holomorphic functions on $X$ as follows: Suppose $f \in C^{\nu}(\mathfrak{W}, \mathcal{O})$. If $f_{i_{0} \cdots i_{\nu}}=\sum_{\mu=-\infty}^{\infty} h_{\mu}^{\left(i_{0}, \cdots, i_{\nu}\right)} z_{i}^{\mu}$ is the Laurent series expansion of $f_{i_{0}} \cdots i_{\nu}$ in $z_{i}$ (where $z_{i}$ is the $i$ th coordinate function of $\left.U^{N}\right)$, then $\left(e_{i}(f)\right)_{i_{0}} \cdots i_{\nu}=\sum_{\mu=0}^{\infty} h_{\mu}^{\left(i_{0}, \cdots, i_{\nu}\right)} z_{i}^{\mu}$. By applying Lemma 1 with $X$ replaced by the product of $X$ and $U^{N-1}$, we have $\left\|\left(e_{i}(f)\right)_{i_{0} \cdots i_{\nu}}\right\|_{W i_{0} \cdots i_{\nu}} \leqq \rho\left\|f_{i_{0} \cdots i_{\nu}}\right\|_{W i_{0} \ldots i_{\nu}}$. Observe that $\left(\left(1-e_{i}\right)(f)\right)_{i_{0} \cdots i_{\nu}}$ $=0$ if $i \neq i_{0}, \cdots, i_{\nu}$. For $0 \leqq \nu<N-1$ we have $\left(1-e_{1}\right) \circ\left(1-e_{2}\right) \circ \cdots$ $\circ\left(1-e_{N}\right)=0$ on $C^{\nu}(\mathfrak{W}, \mathcal{O})$, because for any $1 \leqq i_{0}, \cdots, i_{\nu} \leqq N$ there exists $1 \leqq i \leqq N$ such that $i \neq i_{0}, \cdots, i_{\nu}$. Since $e_{i}$ commutes with $\delta$, for $1 \leqq \nu<N$ we have $\left(1-e_{1}\right) \circ\left(1-e_{2}\right) \circ \cdots \circ\left(1-e_{N}\right)=0$ on $B^{\nu}(\mathfrak{W}, \mathcal{\theta})$.

Next we define for $1 \leqq i \leqq N$ and $1 \leqq \nu<N$ a linear map $k_{i}: C^{\nu}(\mathfrak{W}, \mathcal{\theta})$ $\rightarrow C^{\nu-1}(\mathfrak{B}, \mathcal{\theta})$ over the ring of all holomorphic functions on $X$ as follows: If $f \in C^{\nu}(\mathfrak{W}, \mathcal{O})$, then set $\left(k_{i}(f)\right)_{i_{0} \cdots i_{\nu-1}}$ to be the holomorphic function on $W_{i_{0} \cdots i_{\nu-1}}$ whose restriction to $W_{i_{i_{\nu-1}}}$ is $\left(e_{i}(f)\right)_{i_{i_{\nu-1}}}$. Straightforward computation shows that for $1 \leqq i \leqq N$ and $1 \leqq \nu<N$ we have $e_{i}=\delta k_{i}-k_{i} \delta$ on $C^{\nu}(\mathfrak{W}, \mathcal{O})$. Hence for $1 \leqq \nu<N$ we have $\left(1-\delta k_{1}\right) \circ\left(1-\delta k_{2}\right) \circ \cdots \circ\left(1-\delta k_{N}\right)=0$ on $B^{\nu}(\mathfrak{W}, \mathcal{O})$. For $1 \leqq \nu<N$ 
define $\phi_{\nu}: B^{\nu}(\mathfrak{W}, \mathcal{O}) \rightarrow C^{\nu-1}(\mathfrak{W}, \mathcal{O})$ by

$$
\phi_{\nu}=\sum_{\mu=1}^{N}(-1)^{\mu-1} \sum_{i_{1}<\ldots<i_{\mu}} k_{i_{1}} \delta k_{i_{2}} \cdots \delta k_{i \mu} .
$$

Then $\phi_{\nu}$ satisfies the requirement.

Q.E.D.

REMARK. By using sup $\left|\operatorname{Re} f_{i_{0}} \ldots i_{\nu}\right|$ on $W_{i_{0} \cdots i_{\nu}}$ and $\sup \left|\operatorname{Re} \phi_{\nu}(f)_{i_{0} \cdots i_{\nu-1}}\right|$ on $W_{i_{0} \cdots i_{\nu-1}}$ instead of using $\left\|f_{i_{0} \cdots i_{\nu}}\right\|_{W i_{0} \cdots i_{\nu}}$ and $\left\|\phi_{\nu}(f)_{i_{0} \cdots i_{\nu-1}}\right\|_{W i_{0} \cdots i_{\nu-1}}$, a theorem similar to Theorem 3 can be proved. We need only prove a lemma which corresponds to Lemma 1 but uses sup norms of the real parts instead. To do this, we observe that $f \mapsto \operatorname{Re} f$ defines a continuous $R$-linear injection with closed image from the Fréchet space $E$ of all holomorphic functions on $Q$ whose constant coefficients in the Laurent series expansions are real to the Fréchet space of all harmonic functions on $Q$. Hence, for $r<a<b<1$, there exists a constant $C$ such that, if $f \in E$ and $\sup |\operatorname{Re} f| \leqq K$, then $|f(z)| \leqq C K$ on $a \leqq|z| \leqq b$. The desired lemma follows from an argument analogous to the proof of Lemma 1 , but this time we leave $a$ and $b$ fixed instead of letting $a \rightarrow r$ and $b \rightarrow 1$ and do not restrict $|z|$ to $(a, b)$.

Proof of Theorem 1. By Cartan's Theorem B there is a holomorphic function $\tilde{f}$ on $U^{N}$ such that $Z(\tilde{f})=V$. We can assume $V \cap\left(Q_{r^{\prime}}\right)^{N}=\varnothing$ for some $r^{\prime}<r$. We are going to prove $(1)_{k}$ by induction on $k$.

On $U^{k} \times Q^{N-k}$ (and likewise on products obtained by permut$(1)_{k}$ ing the $N$ factors) we can construct a bounded holomorphic function $f^{(k)}$ such that $Z\left(f^{(k)}\right)=\left(U^{k} \times Q^{N-k}\right) \cap V$ and $\left(f^{(k)}\right)^{-1}$ is bounded on $Q^{N}$.

$Q^{N} \cap V=\varnothing$ implies that $\left(U \times Q^{N-1}\right) \cap V$ is an analytic cover over $Q^{N-1}$ of, say, $n$ sheets. There exists a proper subvariety $A$ in $Q^{N-1}$ and locally defined holomorphic functions $g^{(1)}, \cdots, g^{(n)}$ on $Q^{N-1}$ such that $\left(U \times\left(Q^{N-1}-A\right)\right) \cap V=\left\{\left(z_{1}, \cdots, z_{N}\right) \in U \times\left(Q^{N-1}-A\right) \mid z_{1}\right.$ $=g^{(i)}\left(z_{2}, \cdots, z_{N}\right)$ for some $\left.i\right\}$. The bounded holomorphic extension $f^{(1)}$ on $U \times Q^{N-1}$ of $\prod_{i=1}^{n}\left(z_{1}-g^{(i)}\left(z_{2}, \cdots, z_{N}\right)\right)$ satisfies $Z\left(f^{(1)}\right)$ $=\left(U \times Q^{N-1}\right) \cap V$ and $\left(f^{(1)}\right)^{-1}$ is bounded on $Q^{N}$. (1) is proved. Suppose $(1)_{k}$ is true for $1 \leqq k<m$. Then for $1 \leqq i \leqq m$ we can construct a bounded holomorphic function $f_{i}$ on $G_{i}=U^{i-1} \times Q \times U^{m-i} \times Q^{N-m}$ such that $Z\left(f_{i}\right)=G_{i} \cap V$ and $f_{i}^{-1}$ is bounded on $Q^{N}$. By replacing $f_{i}$ by the product of $f_{i}$ with suitable powers of $z_{i}, z_{m+1}, \cdots, z_{N}$, we can assume that we can select a regular branch $h_{i}$ of $\log \left(\tilde{f} / f_{i}\right)$ on $G_{i}$. Since $h_{i}-h_{j}$ $=$ a branch of $\log \left(f_{j} / f_{i}\right)$ has bounded real part on $G_{i} \cap G_{j}$, by the Remark following Theorem 3 we can construct holomorphic functions 
$\tilde{h}_{i}$ on $G_{i}$ with bounded real parts such that $\tilde{h}_{i}-\tilde{h}_{j}=h_{i}-h_{j}=$ a branch of $\log \left(f_{j} / f_{i}\right)$. The holomorphic function $f^{(m)}$ on $U^{m} \times Q^{N-m}$ which agrees with $f_{i} \exp \left(\tilde{h}_{i}\right)$ on $G_{i}$ satisfies $Z\left(f^{(m)}\right)=\left(U^{m} \times Q^{N-m}\right) \cap V$ and is bounded. Moreover, $\left(f^{(m)}\right)^{-1}$ is bounded on $Q^{N}$. (1) $m$ is proved. The theorem follows from $(1)_{N}$.

Q.E.Di

Proof of TheOREM 2. By Theorem 1 we can construct $g \in H^{\infty}\left(U^{N}\right)$ such that $Z(g)=V$. The construction implies that $g^{-1}$ is bounded on $Q^{N}$. Take $f \in H^{\infty}(V)$. By Cartan's Theorem B $f$ is the restriction to $V$ of a holomorphic function $\bar{f}$ on $U^{N}$. We are going to prove $(2)_{k}$ by induction on $k$.

On $U^{k} \times Q^{N-k}$ (and likewise on products obtained by permut(2) ${ }_{k} \quad$ ing the $N$ factors) we can construct a bounded holomorphic function $f^{(k)}$ which agrees with $f$ on $\left(U^{k} \times Q^{N-k}\right) \cap V$.

From the conditions of special Rudin subvarieties we conclude that $\left(U \times Q^{N-1}\right) \cap V$ is an unbranched analytic cover over $Q^{N-1}$ of, say, $n$ sheets. There are locally defined holomorphic functions $g^{(1)}, \ldots$, $g^{(n)}$ on $Q^{N-1}$ such that $\left(U \times Q^{N-1}\right) \cap V=\left\{\left(z_{1}, \cdots, z_{N}\right) \in U \times Q^{N-1} \mid z_{1}\right.$ $=g^{(i)}\left(z_{2}, \cdots, z_{N}\right)$ for some $\left.i\right\}$. The function $f^{(1)}\left(z_{1}, \cdots, z_{N}\right)$ $=\sum_{i=1}^{N} f\left(g^{(i)}\left(z_{2}, \cdots, z_{N}\right), z_{2}, \cdots, z_{N}\right)\left(\prod_{j \neq i, 1 \leq j \leq n}\left(z_{1}-g^{(j)}\left(z_{2}, \cdots, z_{N}\right)\right)\right)$ $\left(\prod_{j \neq i, 1 \leq j \leq n}\left(g^{(i)}\left(z_{2}, \cdots, z_{N}\right)-g^{(j)}\left(z_{2}, \cdots, z_{N}\right)\right)\right)^{-1}$ is well defined, agrees with $f$ on $\left(U \times Q^{N-1}\right) \cap V$, and is bounded. (2) ${ }_{1}$ is proved. Suppose $(2)_{k}$ is true for $1 \leqq k<m$. We can construct bounded holomorphic functions $f_{i}$ on $G_{i}=U^{i-1} \times Q \times U^{m-i} \times Q^{N-m}, 1 \leqq i \leqq m$, such that $f_{i}=f$ on $G_{i} \cap V$. Let $h_{i}=\left(\tilde{f}-f_{i}\right) / g$ on $G_{i}$. Since $h_{i}-h_{j}=\left(f_{j}-f_{i}\right) / g$ is bounded on $G_{i} \cap G_{j}$ (because $g^{-1}$ is bounded on $Q^{N}$ ), we can construct by Theorem $3 \tilde{h}_{i} \in H^{\infty}\left(G_{i}\right)$ such that $\tilde{h}_{i}-\tilde{h}_{j}=h_{i}-h_{j}=\left(f_{j}-f_{i}\right) / g$. The holomorphic function $f^{(m)}$ on $U^{m} \times Q^{N-m}$ which agrees with $f_{i}+g \tilde{h}_{i}$ on $G_{i}$ is bounded and agrees with $f$ on $\left(U^{m} \times Q^{N-m}\right) \cap V$. (2) $)_{m}$ is proved. By $(2)_{N}$ we can construct $f^{(N)} \in H^{\infty}\left(U^{N}\right)$ which agrees with $f$ on $V$. It is clear from the constructions that the map defined by $f \mapsto f^{(N)}$ is a bounded linear map from $H^{\infty}(V)$ to $H^{\infty}\left(U^{N}\right)$.

Q.E.D.

The author wishes to thank $H$. Alexander for pointing out some errors in an earlier version of this note.

\section{REFERENCES}

1. H. Alexander, Extending bounded holomorphic functions from certain subvarieties of a polydisc, (to appear).

2. W. Rudin, Zero-sets in polydiscs, Bull. Amer. Math. Soc. 73 (1967), 580-583.

UNIVERSITY OF Notre DAME 